\title{
Processo ensino-aprendizagem no campo da organização da informação
}

Teaching-learning process in the field of information organization

Proceso de enseñanza y aprendizaje en el ámbito de la organización de información

Felipe Mozart de Santana NASCIMENTO ${ }^{1}$

Correspondência

Autor para correspondência: Felipe Mozart de Santana Nascimento Endereço completo: Avenida Um, bl. 41, apto. 407, Curado IV - Jaboatão dos Guararapes, Pernambuco. CEP 54270-090 E-mail: mozart.felipe@gmail.com ORCID: ORCID: https://0000-0002-11348147

\section{(c) (i) (9)}

Submetido em: 01/12/2019

Aceito em: 02/01/2020

Publicado em: 22/05/2020

1 Doutorando em Ciência da Informação pela Universidade Federal de Pernambuco. Pesquisador Bolsista da Fundação de Amparo à Ciência e Tecnologia de Pernambuco. 


\section{RESUMO}

Objetiva apresentar o percurso didático do processo ensino-aprendizagem na disciplina Organização da Informação em uma turma de pós-graduação lato sensu em Gestão de Unidades de Informação. Reflete a prática de ensino nas instâncias bases da área de Organização da Informação, no campo da Ciência da Informação. Justifica-se pela discussão e relevância didática aplicadas no processo de formação dos discentes, desenvolvendo habilidades e competências no exercício profissional. Caracterizado como um estudo do tipo documental, empírico qualitativo e descritivo interpretativo, a pesquisa apresenta como resultado um mapeamento do processo de ensino-aprendizagem da disciplina em questão. Os resultados apoiam-se em três eixos fundamentais que descrevem: a) a proposta do curso de especialização em Gestão de Unidades de Informação para a formação do Especialista Gestor de UIs; b) a proposta da disciplina Organização da Informação, considerando o plano de ensino bem como seus elementos constituintes, como ementa e objetivo; e, por fim, o mapeamento do percurso didático. Colabora fortemente com o campo da Educação em Ciência da Informação ao introduzir aspectos pedagógicos como a Metodologia da Problematização com o Arco de Maguerez, uma vez que este tipo de metodologia parte da observação do recorte da realidade associando-a ao tema pertinente de estudo. $O$ uso e aplicação do Arco de Maguerez é percebido como um caminho metodológico voltado à formação profissional, e ao ser explorado neste estudo apoiou-se nos interesses do desenvolvimento e exercício do especialista.

Palavras-chave: Ciência da Informação. Formação profissional. Percurso Didático. Processo ensino-aprendizagem. Organização da Informação.

\section{ABSTRACT}

To present the didactic course of the teaching-learning process in the discipline of Information Organization in a lato sensu postgraduate class in Management of Information Units. It reflects the practice of teaching based on the area of Information Organization, in the field of Information Science. Justification for the discussion and didactic relevance applied in the training process of students, developing the skills in the professional practice. Characterized as a documental, qualitative and descriptive interpretative study, the research presents as a result a mapping of the teaching-learning process of the discipline. The results are based on three fundamental axes that describe: a) the proposal of the specialization course in management of information units for the training of the specialist UIs manager; b) The proposal of the discipline Information Organization, considering the teaching plan as well as its constituent elements, namely: menu and objective; and, finally, the mapping of the didactic path. It collaborates much with the field of education in information science by introducing pedagogical aspects such as the methodology of problematization with the Maguerez Arch, since contradictory observation of the clipping of reality associating it with the pertinent theme of Study. The use and application of the arch is perceived 
as a methodological path focused on professional training, in which its proper insertion in this study is supported by the interests of the development and exercise of the specialist.

Keywords: Didactic course. Information Organization. Information Science. Teaching-learning process. Vocational training.

\section{RESUMEN}

Su objetivo es presentar el camino didáctico del proceso de enseñanzaaprendizaje en la disciplina Organización de la información en una clase de posgrado lato sensu en Gestión de unidades de información. Refleja la práctica docente en las instancias base del área de Organización de la Información, en el campo de las Ciencias de la Información. Está justificado por la discusión y la relevancia didáctica aplicada en el proceso de capacitación de los estudiantes, el desarrollo de habilidades y competencias en la práctica profesional. Caracterizada como un estudio documental, empírico, cualitativo y descriptivo, la investigación presenta como resultado un mapeo del proceso de enseñanza-aprendizaje de la asignatura en cuestión. Los resultados se basan en tres ejes fundamentales que describen: a) la propuesta del curso de especialización en Gestión de Unidades de Información para la formación del Gerente Especialista de IU; b) la propuesta de la organización de información disciplinaria, considerando el plan de enseñanza así como sus elementos constitutivos, tales como menú y objetivo; y, finalmente, el mapeo de la ruta didáctica. Colabora fuertemente con el campo de la Educación en Ciencias de la Información mediante la introducción de aspectos pedagógicos como la Metodología de establecimiento de problemas con el Arco de Maguerez, ya que este tipo de metodología parte de la observación del corte de la realidad y lo asocia al tema de estudio relevante. El uso y la aplicación del Arco de Maguerez se percibe como una ruta metodológica dirigida a la formación profesional, y cuando se explora en este estudio, se basa en los intereses del desarrollo y el ejercicio del especialista.

Palabras clave: Ciencias de la información. Formación profesional. Ruta didáctica. Proceso de enseñanza-aprendizaje. Organización de la información.

\section{INTRODUÇÃO}

As técnicas de planejamento e execução de um plano de ensino requer atenção quanto à ementa e aos objetivos que se desejam cumprir. Em consonância a isto, o percurso didático a ser trilhado surge como um desafio a ser explorado pelo docente, quanto à determinação do processo de ensino, e pelos 
discentes, quanto à reflexão do processo de aprendizagem.

Nesse sentido, esta pesquisa vislumbra a caracterização do percurso didático de ensino-aprendizagem explorado em uma turma de pós-graduação lato sensu (especialização) em Gestão de Unidades de Informação. A especialização surgiu com a proposta de qualificação profissional para atuar no campo da gestão de unidades de informação, estruturando-se por meio da construção do conhecimento teórico-prático com foco no exercício da prática profissional. Entre os componentes curriculares a disciplina Organização da Informação (OI) integra o eixo básico do curso e é tida como objeto de estudo desta pesquisa.

Este estudo justifica-se pelas discussões, pelo caráter e relevância didática aplicados em uma turma de especialização, especificamente na disciplina Organização da Informação, a qual buscou-se desenvolver habilidades e competências profissionais dos discentes envolvidos. O processo ensinoaprendizagem apoiou-se na resolução de problemas reais encontrados nas instituições de trabalho dos discentes e como método para resolução dos problemas encontrados adotou-se a Metodologia da Problematização baseada no Arco de Maguerez.

Desta forma, a pesquisa objetiva apresentar o percurso didático de ensino-aprendizagem na disciplina "Organização da Informação" em uma turma de pós-graduação lato sensu (especialização) em Gestão de Unidades Informação.

Este estudo está estruturado por esta seção introdutória e outras quatro seções: (II) revisão bibliográfica 
sobre a disciplina alvo desta pesquisa, considerando os estudos de Brascher e Café (2008), Aguiar e Kobashi (2013), Vieira e Pinho (2015), Pando (2018) e Braz, Nascimento e Feitoza (2018); (III) a orientação metodológica do estudo baseada, principalmente, em Berbel (2012) e Bordenave (1998); (IV) análise do processo ensino-aprendizagem; e, por fim, (V) as considerações finais.

\section{ORGANIZAÇÃO DA INFORMAÇÃO}

A OI é vista como uma das disciplinas de estudos da Ciência da Informação (CI) por integrar um eixo de processos como coleta, processamento, disseminação, recuperação e uso da informação. Esses processos evoluem de acordo com o progresso e desenvolvimento social, os quais destacam-se as contribuições da tecnologia da informação e comunicação bem como sua constante atualização (BRAZ; NASCIMENTO; FEITOZA, 2018, p. 68). Corroboram, neste sentido, Aguiar e Kobashi (2013, p. 6) ao perceberem a OI "como uma série de atividades processuais com a finalidade de descrever intelectualmente conteúdos documentais para serem representados nos sistemas de recuperação da informação". Ainda neste contexto,

Organização da Informação é uma das atividades de maior importância por ter uma atuação mediadora entre as informações e o atendimento às necessidades de informações dos mais diferentes usuários, razão pela qual passamos a discutir historicamente sua constituição enquanto espaço que fornece métodos e estratégias para lidar com o crescente volume informacional (PANDO, 2018, p. 147). 
Enquanto atividade, a OI envolve dois exercícios comuns, tais como: a descrição física e a descrição de conteúdo dos objetos informacionais. A integração dessas atividades resulta na Representação da Informação (RI), tida como produto deste processo. Logo, entende-se como RI um conjunto de elementos descritivos que representam os atributos de um objeto informacional específico (BRASCHER; CAFÉ, 2008, p. 5).

Assim sendo, Pando (2018) desenvolveu um mapa operacional das atividades pertinentes à OI, enquanto campo de estudo da CI, conforme Figura 1:

Figura 1 - Operações do campo de Organização da Informação 


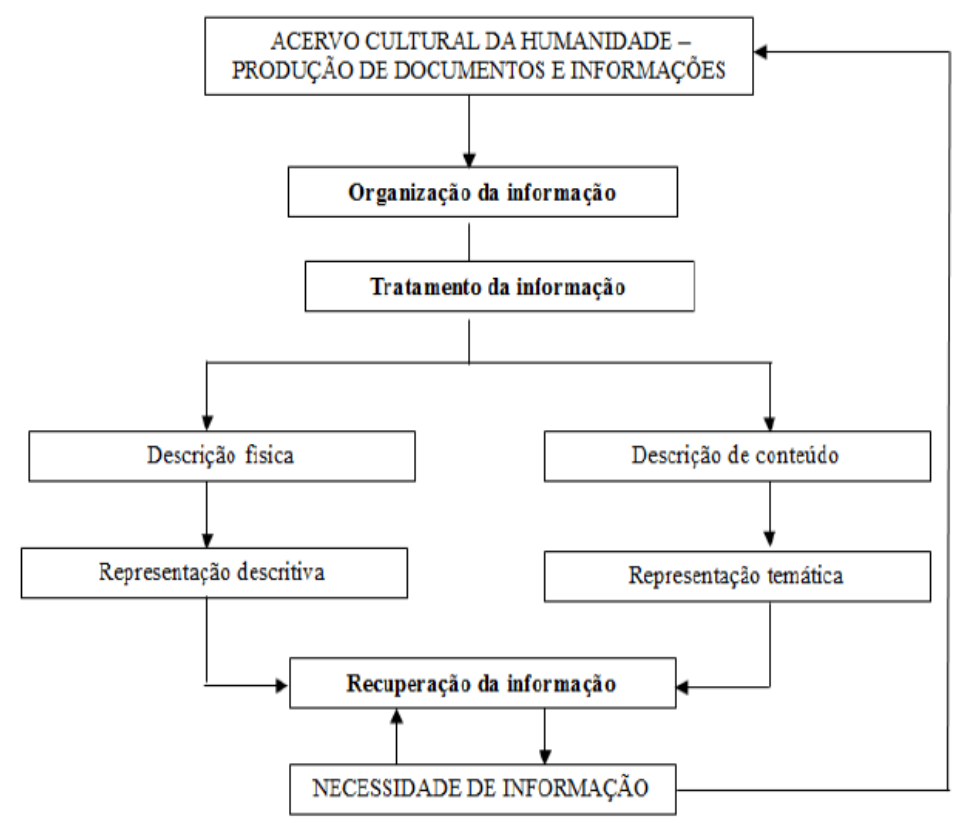

Fonte: Pando (2018, p. 150).

De acordo com o mapeamento do ciclo operacional das atividades de OI, é perceptível a relevância de estudo deste campo e sua importância para os sistemas de recuperação de informação (SRI) ao considerar que a prática assertiva atua diretamente na recuperação da informação e atende prontamente as necessidades dos usuários de um sistema de informação (PANDO, 2018).

Ainda por meio das operações da figura 1, percebe-se que a OI "exerce uma atividade nuclear enquanto mediadora, sendo responsável por estabelecer uma ponte entre a representação e o uso da informação" e que, quanto às suas funções, busca "organizar registros de informação para arranjá-los sistematicamente, de forma a possibilitar sua recuperação e posterior uso" (VIEIRA; PINHO, 2015, p. 112). Fundamental destacar que se somam às operações acima outras atividades para OI como classificação, catalogação, indexação, 
sistematização e disponibilização.

Contextualizou-se nesta seção o viés relacional entre a percepção da OI enquanto campo de estudo e enquanto processos operacionais na perspectiva da Ciência da Informação. A integração dessas percepções nos permite compreender o exercício profissional do Gestor de uma unidade de informação quanto ao exercício operacional a ser desenvolvido no ambiente de atuação.

\section{METODOLOGIA}

A classificação metodológica desta pesquisa pauta-se como um estudo de caráter documental e empírico qualitativo, descritivo-interpretativo em que se apoiam nos pontos abaixo:

I) Compreensão da proposta da especialização e do profissional Gestor de Unidades de Informação, tendo como elemento de consulta o Projeto Pedagógico do Curso (PPC).

II) O apoio empírico envolve a primeira turma (2018) com uma estimativa de 15 alunos matriculados na disciplina de Organização da Informação ministrada no primeiro semestre de 2018.

Adotou-se como apoio à resolução dos estudos de casos reais a Metodologia da Problematização com o Arco de Maguerez. Em linhas gerais, conforme aponta Berbel (2012, p. 71), a Metodologia da Problematização "é uma das manifestações do construtivismo pedagógico. Como tal, partilha com outros métodos construtivistas alguns princípios fundamentais". Esses princípios são elencados por Berbel 
(2012), baseada em Bordenave (1998, p. 7), conforme descritos abaixo:

- Parte-se da realidade com a finalidade de compreendê-la e de construir conhecimento capaz de transformá-la;

- Utiliza-se o que já se sabe sobre a realidade (conteúdos), não como algo absoluto e definitivo nem como um fim em si mesmo, mas como subsídio para encontrar novas relações, novas "verdades", novas soluções;

- Os protagonistas da aprendizagem são os próprios aprendentes, por isso acentua-se a descoberta, a participação na ação grupal, a autonomia e a iniciativa;

- Desenvolve-se a capacidade de perguntar, consultar, experimentar, avaliar, características da consciência crítica.

Ao que relaciona a Metodologia da Problematização com o Arco de Maguerez, Berbel (2012) caracteriza essa relação citando Bordenave (1998, p. 7).

Como todos os métodos construtivistas, a Metodologia da Problematização em geral, e o Método do Arco de Maguerez, em particular, partilham a mesma sequência epistemólogica com três importantes processos sociais: o processo da pesquisa, o processo do planejamento e o processo da solução de problemas. Com efeito, todos esses processos parte de uma situação que provoca questionamentos. Em resposta a eles configura-se um quadro conceitual que permite a análise teórica, formulam-se hipóteses orientadoras, colhem-se dados relevantes e chega-se a uma síntese ou solução que envolve algum tipo de transformação da realidade (grifos do autor).

A congregação dos elementos chave apresentados nesta seção permitiu a apresentação do percurso ensino aprendizagem na disciplina "Organização da Informação" em 
uma turma da pós-graduação lato sensu (especialização) em Gestão de Unidades Informação. Essa caracterização está descrita na seção a seguir.

\section{RESULTADOS}

$\mathrm{Na}$ proposta de cumprir o objetivo deste estudo, esta seção está estruturada nos seguintes pontos: compreensão da proposta do curso para formação do Especialista Gestor de Unidades de Informação, a caracterização da disciplina Organização da Informação e por fim, apresentar o percurso didático de ensino-aprendizagem promovido pela disciplina.

a) Proposta do curso para formação do Especialista Gestor de Unidades de Informação.

A especialização em Gestão de Unidades de Informação é ofertada para atender um carecimento de formação profissional para os indivíduos que trabalham ou pretendem exercer atividades profissionais em unidades de informação, atendendo a carência de especialistas em organização e gestão de acervos. É destinada à profissionais das áreas de Administração, Arquivologia, Biblioteconomia, Gestão da Informação, Gestão de Recursos Humanos, Museologia e áreas afins. Cumpre uma carga horária de 360 horas com duração de 18 meses. Entre os componentes curriculares encontram-se disciplinas voltadas à conservação de unidades de informação, ciência da informação, políticas públicas de informação, gestão documental, práticas de exposições, acessibilidade em unidades de informação, educação patrimonial e organização da 
informação. Esta última está descrita a seguir.

b) Proposta da disciplina Organização da Informação.

A disciplina tem como ementa os princípios, modelos, técnicas e métodos orientados à organização e representação da informação registrada em quaisquer suportes informacionais, sob a ótica da Ciência da Informação e buscou atender ao objetivo de compreender os processos de Organização da Informação, considerando seus aspectos teóricos e práticos no âmbito da Ciência da Informação e sua característica interdisciplinar. O conteúdo programático alicerçou-se em seis encontros, dividindo-se em:

I. Bases epistemológicas da Organização e Representação da Informação;

II. Conceito na Organização e Representação da Informação;

III. Sistemas de Organização do Conhecimento: classificação, taxonomia e tesauros;

IV.Sistemas de Organização do Conhecimento: ontologias e mapas conceituais;

V. Seminários de Práticas em Organização da Informação.

As estratégias dos encontros foram dispostas conforme cronograma oficial do curso, totalizando uma carga horária global de 24 horas, distribuídas em aulas expositivas dialogadas utilizando leituras de textos, debates, estudos dirigidos, atividades práticas, preparação e apresentação de um estudo de caso real. 
c) O percurso ensino-aprendizagem na disciplina

O percurso de ensino-aprendizagem envolveu o docente responsável pela disciplina e os discentes matriculados na disciplina. Neste percurso foram explorados os processos ilustrados abaixo com a finalidade de desenvolver habilidades e competências que cooperam para a formação do especialista:

Figura 2 - Processo ensino-aprendizagem na disciplina Organização da Informação

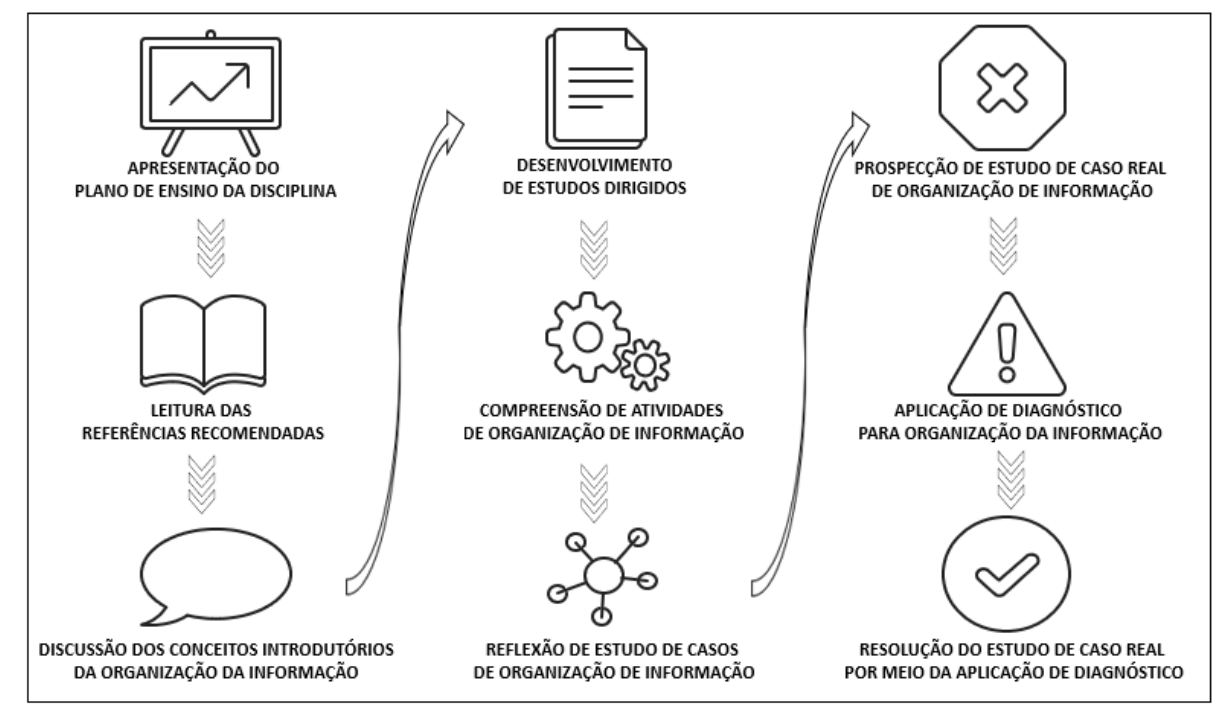

Fonte: elaborado pelo autor.

Conforme pode ser visualizado na Figura 2, o processo ensino-aprendizagem iniciou-se na apresentação do plano de ensino em sala de aula. Definido por Nascimento (2010, p. 2), o plano de ensino "é um recurso utilizado pelo professor para sistematizar as intenções e finalidades de sua ação educativa na prática pedagógica cotidiana". Apresentar o plano, socializando-o no primeiro dia de aula, torna o processo de aprendizagem elucidativo ao discente, internalizando este sujeito ao processo de ensino.

Por meio do plano foram apresentadas as referências bases e complementares para a disciplina, conforme o quadro 1: 
Quadro 1 - Referências apresentadas no plano de ensino da disciplina.

CAFÉ, L. ; BRASCHER, M. Organização da informação ou organização do conhecimento? In: ENCONTRO NACIONAL DE PESQUISA EM CIÊNCIA DA INFORMAÇÃO (ENANCIB), 9., 2008, São Paulo. Anais [...] São Paulo, USP, 2008.

CAFÉ, L. et al. Análise conceitual do termo organização do conhecimento em teses brasileiras. Revista Brasileira de Biblioteconomia e Documentação, v. 13, n. 1, 2017. MOREIRO GONZÁLEZ, J. A. Linguagens documentárias e vocabulários semânticos para a web. Elementos conceituais. Salvador de Bahia: EDUFBA, 2011.

NASCIMENTO, F. M. S.; PINHO, F. B. A. Ontologia na gestão de conhecimento jurídico. Revista P2P e INOVAÇÃo, v. 4, n. $2,2018$.

OTTONICAR, S. L. C.; SANTOS, B. R. P.; MORAES, I. S. Aplicabilidade da competência em informação e da organização do conhecimento no processo de gestão da informação. Revista Digital de Biblioteconomia \& Ciência da Informação, v. 15, n. 3, 2017.

PINHO, F. A. Fundamentos da organização e representação do conhecimento. Recife: Editora da UFPE, 2009.

RAMALHO, R. R. A. S. O modelo de dados skos: novas perspectivas no âmbito da representação do conhecimento. In: ENCONTRO NACIONAL DE PESQUISA EM CIÊNCIA DA INFORMAÇÃO, 17., 2016. Anais[...] São Paulo: ANCIB, 2016. ROBREDO, J. Organização dos documentos ou organização da informação: uma questão de escolha. DataGramaZero, v.5, n.1, fev. 2004.

VITAL, L. P.; MEDEIROS, G. M.; BRÄSCHER, M. Classificação e descrição arquivística como atividades de organização e representação da informaçÃo e do conhecimento. Brazilian Journal of Information Science, v. 11, n. 4, 2017.

Fonte: elaborada pelo autor.

Os textos indicados na leitura das referências recomendadas buscaram facilitar o entendimento do campo e promover uma discussão introdutória dos conceitos de 
organização da informação, como Café e Brascher (2008), Café (2017), Pinho (2009) e Robredo (2004). A leitura, compreensão e discussão desses elementos introdutórios incentivaram os discentes ao desenvolvimento de estudos dirigidos que, por sua vez, contribuíram para o entendimento base da disciplina, o quadro epistemológico e conceituais da OI.

Em sequência foram explorados textos teóricos e práticos com o propósito de compreender as atividades de OI, bem como seus processos, seus instrumentos e produtos. Além dos textos contidos no quadro de referências, como Moreiro González (2011), Nascimento e Pinho (2018), Ramalho (2016) e Vital, Medeiros e Brascher (2017), os discentes buscaram outros textos do campo de OI e da temática profissional de interesse.

A partir da atividade de reflexão de estudos de casos de organização de informação inseriu-se como apoio ao processo de ensino aprendizagem a Metodologia da Problematização com o Arco de Maguerez. O desenho do Arco está representado na figura 3, a seguir:

Figura 3: Arco de Maguerez

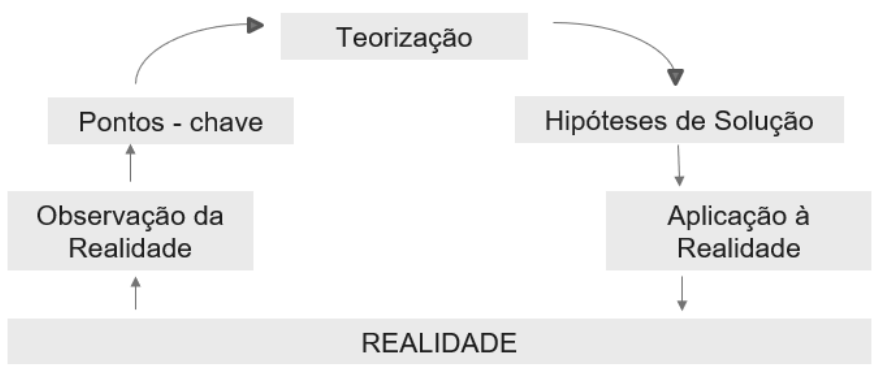

Fonte: Adaptado de Bordenave e Pereira (1982, p. 10) 
A compreensão e aplicabilidade da Metodologia da Problematização e Arco de Maguerez podem ser apoiadas na descrição de Berbel (2012, p. 15):

[...] as ações para o desenvolvimento do processo partem da observação de um recorte da realidade associado à temática eleita para o estudo. Da observação analítica e crítica dessa parcela da realidade é extraído um problema relevante para o estudo. Seguem-se as definições dos aspectos do problema a estudar, o estudo propriamente dito de tais aspectos, que preparam ara as últimas etapas, das hipóteses de solução e da aplicação de uma ou mais dessas hipóteses na realidade da qual se extraiu o problema.

\section{A prospecção de estudo de caso real de organização} de informação envolveu o ambiente de trabalho dos discentes envolvidos. A teoria aprendida em sala de aula somada às reflexões de estudos de casos já desenvolvidos, facilitaram o processo de reconhecimento de carências nas atividades de organização da informação nas unidades em que os discentes exerciam suas práticas profissionais, motivando-os à aplicação de diagnóstico para essas atividades. Esses pontos resultaram em projetos reais, como por exemplo o projeto intitulado "Organização da Informação em Unidades de Informação: diagnósticos em bibliotecas" cujo objetivo foi o de "criar uma política de indexação que auxilie a melhoria no processo de recuperação da informação nos sistemas das bibliotecas alvos de estudo", além de outro projeto intitulado "Processos de Organização da Informação: um estudo de caso do repositório 
do Instituto de Tecnologia de Pernambuco (ITEP)"2 em que objetivou "propor processos de organização da informação para o planejamento do repositório do ITEP".

A última atividade do processo de ensino aprendizagem diz respeito ao produto gerado por meio da integração de todas as etapas do processo, em que se firma na resolução do estudo de caso real por meio da aplicação de diagnósticos, ao qual se concretizou todo aprendizado em sala de aula e aplicação do conhecimento aprendido nos ambientes de trabalhos.

\section{CONSIDERAÇÕES FINAIS}

É papel do docente, por meio de suas práticas de ensino, perceber 0 aluno como um agente em potencial e transformador da realidade em que atua. Essa percepção deve estar aliada ao processo de aprendizagem do indivíduo. Integra-se ao processo de ensino e aprendizagem os moldes políticos e sociais dos ambientes que prospectam, acolhem e desenvolvem esses sujeitos. Esses ambientes são os lugares em que se cumprem as atividades profissionais e põe em exercício os conhecimentos explorados durante o processo didático concebido em sala.

O mapeamento do processo ensino aprendizagem deste estudo pode ser utilizado por outros perfis, campos ou áreas de

\footnotetext{
2 Para melhor visualização da aplicação da metodologia utilizada baseada na descrição e relato de um estudo real desenvolvida na disciplina, recomenda-se a leitura na íntegra da pesquisa:
} 
conhecimento distintas, estejam elas relacionadas ou não à CI. Ao que diz respeito ao desenvolvimento da disciplina de Organização da Informação na formação do Especialista em Gestão de Unidades de Informação, cumpriu-se com êxito os elementos constituintes do plano de ensino, como a ementa e o objetivo da disciplina. Assim, o alcance do objetivo foi cumprido ao apresentar os pontos, estruturados e descritos na seção anterior, como: a proposta do curso para a formação do profissional em questão, a proposta da disciplina Organização da Informação e, por fim, o percurso ensino-aprendizagem na disciplina.

No sentido de avaliar os aspectos didáticos da disciplina os discentes responderam um questionário aplicado no fim da disciplina, destacando-se como sucesso os seguintes pontos: proporção entre conceitos; exemplos práticos e exercícios; programa da disciplina e bibliografia; aplicação dos novos conhecimentos e habilidades no trabalho e aprendizagem dos temas abordados. Ainda quanto ao fim da disciplina, foi promovido o I Simpósio de Gestão da Informação em que foi organizado e executado pelos discentes por meio da Mostra Pedagógica em Organização da Informação.

\section{REFERÊNCIAS}

AGUIAR, F. L.; KOBASHI, N. Y. Organização e representação do conhecimento: perspectivas de interlocução interdisciplinar entre Ciência da Informação e Arquivologia. In: ENCONTRO NACIONAL DE PESQUISA EM CIÊNCIA DA INFORMAÇÃO, 16., 2013, Florianópolis. Anais[...] Florianópolis: UFSC, 2013. Disponível em: https://bit.ly/2Va00CV Acesso em: 09 mai. 2019. 
Berbel, N. A. N. A Metodologia da Problematização com o Arco de Maguerez: uma reflexão teórico-epistemológica. Londrina: EDUEL, 2012.

BORDENAVE, J. D. Prefácio. In: BERBEL, N. A. N. (Org.). Metodologia da Problematização. Experiências com questões de Ensino Superior. Londrina: EDUEL, 1998.

BORDENAVE, J. D.; PEREIRA, A. M. Estratégias de ensino aprendizagem. 4. ed. Petrópolis: Vozes, 1982.

BRASCHER, M.; CAFÉ, L. Organização da Informação ou Organização do Conhecimento? In: ENCONTRO NACIONAL DE PESQUISA EM CIÊNCIA DA INFORMAÇÃO, 11., 2008, São Paulo. Anais[...] São Paulo: USP, 2008. Disponível em https://bit.ly/2DX7xdI acesso em $07 \mathrm{fev.} 2018$.

BRAZ, M. I.; NASCIMENTO, F. M. S.; FEITOZA, R. B.; Práticas avaliativas no campo da Organização da Informação. ConCI: Conv. Ciênc. Inform., São Cristóvão (Sergipe), v. 1, n. 2, Ed. Especial, p. 66-73, 2018. Disponível em: https://bit.ly/2VhFdc4 Acesso em: 09 maio 2019.

NASCIMENTO, M. J. Planos de ensino de "Usuários da Informação" nos cursos de Biblioteconomia do Brasil. DataGramaZero, v. 10, n. 1, p. 1-12, 2010.

\section{PANDO, D. A. Epistemologia da organização da} informação: uma análise de sua cientificidade no contexto brasileiro. 2018. 463 f. Tese (Doutorado em Ciência da Informação) - Universidade Estadual Paulista (UNESP), Marília, 2018. Disponível em: https://bit.ly/2YezLbC Acesso em: 09 maio 2019.

VIEIRA, J. M. L.; PINHO, F. A. A contribuição da organização e da visualização da informação para os sistemas de recuperação de informação. Informação \& Informação, v. 20, n. 1, p. 110-136, 2015. Disponível em: https://bit.ly/2H8Vyuu Acesso em: 09 maio 2019. 